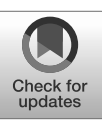

\title{
13 Kreislaufwirtschaft als Säule des EU Green Deal
}

\begin{abstract}
Florian Schaller, Annette Randhahn, Eyk Bösche, Jakob Michelmann
Die Nutzung erneuerbarer Energien und Energieeffizienz sind bislang die zentralen Ansätze zur Minderung der anthropogenen Ursachen des Klimawandels. Zudem übt der weltweite Ressourcenverbrauch enormen Änderungsdruck auf unser bestehendes überwiegend lineares Wirtschaftssystem aus. Um Emissionen und Ressourcenverbrauch weiter zu reduzieren, setzt der im November 2019 von der EU-Kommission vorgestellte Green Deal auf die Etablierung einer Kreislaufwirtschaft. Hat sie das Potenzial, das Paradigma der linearen Wirtschaft abzulösen?
\end{abstract}

Die Wirtschaft der Industrienationen folgt bislang einem linearen Prinzip, nach dem Konsumgüter produziert, genutzt und entsorgt werden (Wilts 2016). Dabei werden überwiegend Primärmaterialien mit energetischem Aufwand gewonnen und in der Produktion veredelt. Die von den Konsument:innen vielerorts geforderte und auch weithin umgesetzte Trennung des Mülls bei der Entsorgung ist der Beginn eines Recyclingprozesses, in dem Abfallprodukte als Sekundärrohstoffe zurück in den Rohstoffkreislauf eintreten. Allerdings werden in einer Gesamtbilanz nur vergleichsweise wenige Stoffe in diesen Wertstoff- und Rohstoffkreislauf zurückgeführt und dies oftmals unter gesundheitlich belastenden Umständen für die beteiligten Arbeitskräfte. ${ }^{75}$ Ein Großteil der Produkte landet abschließend in der Verbrennung, im Meer oder in Deponien, obwohl die Produkte teils wieder genutzt werden könnten. Trotz eines Trennungssystems, auf das wir so stolz sind, sind wir noch weit von einer Kreislaufwirtschaft entfernt.

Die Auswirkungen der linearen Wirtschaft sind tiefgreifend sowohl für die Ökosysteme als auch die Gesellschaft (zum Beispiel die Verschmutzung der Gewässer, der Böden und der Luft) und könnten starke Veränderungen oder gar den Zusammenbruch natürlicher Stoffkreisläufe herbeiführen. Hinzu kommt die Verknappung von Rohstoffen, die Preise steigen lässt und immer wieder Anlass für geopolitische Auseinandersetzungen ist. Traurig ist schließlich auch der Umstand, dass alljährlich der

75 In Deutschland wurden 201537 Prozent der 5,2 Mio. Tonnen ordnungsgemäß entsorgten Kunststoffe als Wertstoff und ein Prozent also Rohstoff wiederverwertet. Die restlichen 62 Prozent der im In- und Ausland sortierten Kunststoffe wurden Deponien zugeführt oder verbrannt (vgl. Deutscher Bundestag 2018:53). 
Tag im Kalender vordatiert werden muss, an dem die Menschheit der Erde mehr Stoffe entnommen hat, als die Natur wiederherstellen kann. ${ }^{76}$

Diese keineswegs nachhaltige Wirtschaftsweise scheint jedoch einer Gesellschaft zu entsprechen, die nach Steigerung des materiellen Wohlstands und schnellem Konsum strebt (vgl. Rosa 1999:155-158). Das dafür entfachte Wirtschaftswachstum profitiert von der Kultur des Wegwerfens. Zum Teil durch das Design bereits induzierte immer kürzere Produktlebenszyklen machen das Wegwerfen geradezu zum Standard im Alltag (Wilts 2016). Der Handel verstetigt solch lineares Wirtschaften, wenn er zum Beispiel die Vermarktung von Produktneuigkeiten in immer kürzeren Abständen anstrebt. So müssen beispielsweise immer wieder bei Verkäufen Benchmarks eingehalten werden (vgl. Dievernich 2012). Solche Entscheidungen können als Lock-in-Effekte eine Pfadabhängigkeit bewirken. Während die Konkurrenz FastConsumer-Goods verkauft, fällt es schwer, dagegenzuhalten und auf Langlebigkeit von Produkten zu setzen.

Das lineare Wirtschaften kann nach Dosi und Perez als technisch-ökonomisches Paradigma einer Wirtschaft charakterisiert werden, das sich als "kollektiv geteilte Logik" (Perez 2009:186) darin manifestiert, dass das gleiche Modell zur Lösung eines Problems angewendet wird (vgl. Dosi 1982). Weiterhin lässt sich mit Dosi (vgl. ebd.) Folgendes nachvollziehen: Wichtige Kriterien für die Herausbildung von Paradigmen sind Vermarktbarkeit, Wirtschaftlichkeit im Sinne von Gewinn und Kostenersparnis, aber auch möglicherweise staatliches Interesse. Paradigmen können für eine bestimmte Zeit eine Innovationslogik für Akteur:innen vorgeben, in diesem Fall Produzieren - Nutzen - Wegwerfen. Es gibt nur zwei Wege, ein Paradigma wie dieses aufzulösen: Entweder wird das Problem obsolet, auf das es sich bezieht - was hier nicht der Fall sein wird. Denn das Problem, das die lineare Wirtschaft bearbeitet, der Bedarf an Verbrauchsgütern, besteht weiterhin. Oder es werden neue Pfade konstituiert und das damit Bestehende wird infrage gestellt.

Tatsächlich treiben Politik, Unternehmen und Organisationen den zweiten Weg in Bezug auf die Linearwirtschaft voran: Ziel ist es, Wirtschaftswachstum und Ressourcenverbrauch zu entkoppeln. Das Modell dafür ist das der Kreislaufwirtschaft. Bei genauerer Betrachtung fällt auf, dass Kreislaufwirtschaft eigentlich gar nichts Neues ist. Sie wurde seit Menschengedenken praktiziert, etwa wenn es darum ging, Steine aus verfallenen Gebäuden in einem neuen Gebäude wiederzuverwenden. Der kreislaufwirtschaftliche Gedanke ruht als ein in der Pfadtheorie sogenannter "schlafender Pfad" (Dievernich 2012), auf den Menschen unter bestimmten Bedingungen Zugriff

76 Während dieser Tag, der sogenannte Earth Overshoot Day, 1987 noch auf den 19.12. fiel, wird dieser immer früher datiert. 2019 war er bereits am 29. Juli (vgl. Umweltbundesamt 2019). 
haben. Dazu ist es allerdings notwendig, soziale, ökonomische und politische Lockin-Effekte zu erkennen und aufzulösen (vgl. Garud 2010).

\section{Von der Linear- zur Kreislaufwirtschaft}

Mit der Circular Economy verbinden sich hohe Erwartungen: Das Modell soll den Spagat ermöglichen, die Umwelt zu schützen und gleichzeitig am Paradigma des Wirtschaftswachstums festzuhalten: Das Wirtschaftswachstum soll vom Ressourcenverbrauch entkoppelt werden, um im Idealfall weniger Ressourcen zu verbrauchen und Abfälle auf ein Minimum zu reduzieren (Bening et al. 2019). Grundlage des aktuellen Verständnisses des kreislauforientierten Wirtschaftens ist das Circular-Economy-Modell der Ellen MacArthur Foundation. Es existieren jedoch auch weitere Ansätze, welche sich insbesondere im Hinblick auf die Rolle biobasierter Kreisläufe und erneuerbarer Energien unterscheiden (Wilts 2016).

2004 führte Gunter Pauli den Begriff der Blue Economy ein. Kern dieses Ansatzes ist die Orientierung an der Natur. Es werden Geschäftsmodelle für Produkte und Dienstleistungen entwickelt, welche die Kaskadenwirtschaft von Ökosystemen zum Vorbild nehmen (Holzinger 2020). Das Konzept Cradle-to-Cradle geht über das derzeitige Verständnis des zirkulären Wirtschaftens hinaus und wurde 2002 von Michael Braungart und William McDonough entwickelt. Hier werden die Materialien und Ressourcen in zwei Kreisläufe unterteilt, den biologischen und den technischen. Ziel ist es, in beiden Kreisläufen alle Materialien ohne negative Umweltauswirkungen dauerhaft in einem Kreislauf zu halten. Idealerweise sollen alle Materialien vollständig abbaubar sein oder vollständig erhalten bleiben und die Stoffqualitäten beim Recycling keinesfalls vermindert werden. Die vollständige Abkehr von fossilen Brennstoffen ist ein weiterer sehr wichtiger Aspekt dieses Konzepts (Wilts 2016). Das Zero-Waste-Konzept repräsentiert eigentlichen keinen eigenständigen neuen Ansatz, sondern kann eher als ein Zielzustand der Kreislaufwirtschaft sowie der anderen beschriebenen Ansätze verstanden werden. Die mittlerweile globale Zero-Waste-Bewegung vereint allerdings sehr unterschiedliche Aspekte, die von der Reduzierung der zu deponierenden Restmüllmengen bis hin zum umfassend abfallvermeidenden Produktdesign reichen (Wilts 2016). Neben der Dekarbonisierung des Wirtschaftens (Zero Emission) setzt die Politik große Hoffnungen in die Kreislaufwirtschaft (Zero Waste). Dies zeigt etwa das 2015 gestartete EU-Programm zur Kreislaufwirtschaft (Holzinger 2020).

Das Prinzip der Circular Economy basiert auf „R-Innovationen“ für die Produkte und Materialien (s. Abb. 13.1). Angefangen von "Reuse" (Wieder- und Weiterverwendung) und "Repair" (Reparatur) über "Refurbish" (Aufarbeitung) bis hin zum "Recycling". „R-Innovationen" sind umweltschonender als Recycling, weil die in den Gütern enthaltenen „grauen Ressourcen” (Material, Energie, CO2-Emissionen, 


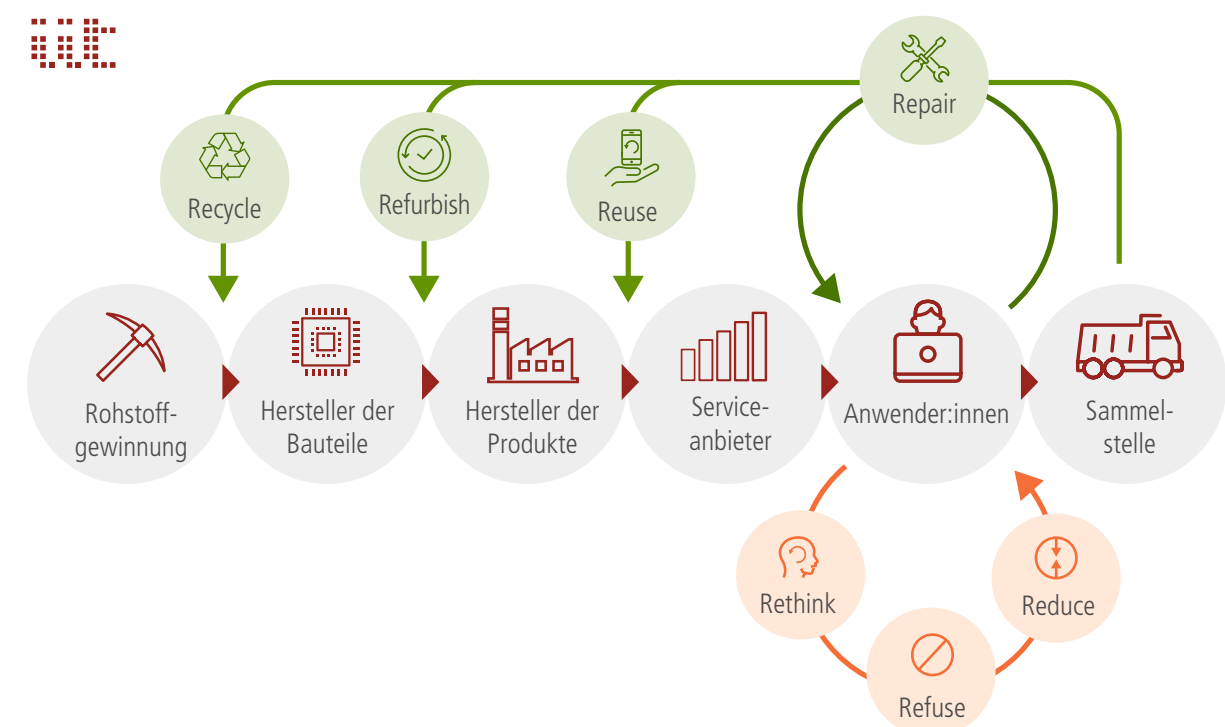

Abb. 13.1 Darstellung des Prinzips der Kreislaufwirtschaft. (Eigene Darstellung nach ifixit und Holzinger 2020; https://store.ifixit.de/pages/kreislaufwirtschaft, zuletzt geprüft am 10.07.2020)

Wasser usw.) weitgehend erhalten bleiben, während ein Recycling nur teilweise und meist unrein jeweils nur die stofflichen Ressourcen zurückgewinnt (Stahel 2020).

Diese am Kreislauf orientierten "R-Innovationen" können auf Seite der Konsument:innen ebenfalls durch vorgelagerte "R-Regeln" im Umgang mit Produkten auf dem Weg zur funktionierenden Circular Economy unterstützt werden. So sollten sich Konsument:innen deutlich stärker damit auseinandersetzen, ob eine geplante Anschaffung tatsächlich notwendig ist (Rethink). Gefolgt von der Verweigerung (Refuse), in einem Zeitalter wachsender Sharing-Modelle alles als Eigentum zu besitzen, bis hin zu der Entscheidung, Produkte zu kaufen, die weniger Ressourcen verbrauchen (Reduce) (Holzinger 2020).

\section{Potenziale der Kreislaufwirtschaft}

Die EU-Kommission knüpft hohe Erwartungen an die vollständige Etablierung der Kreislaufwirtschaft. Sie soll den Druck auf die natürlichen Ressourcen verringern, eine Voraussetzung dafür sein, das Ziel der Klimaneutralität bis 2050 zu erreichen, sowie dem Verlust an biologischer Vielfalt Einhalt gebieten. Zudem soll sie sich positiv auf das BIP-Wachstum und die Schaffung von Arbeitsplätzen auswirken. Die Kommission 


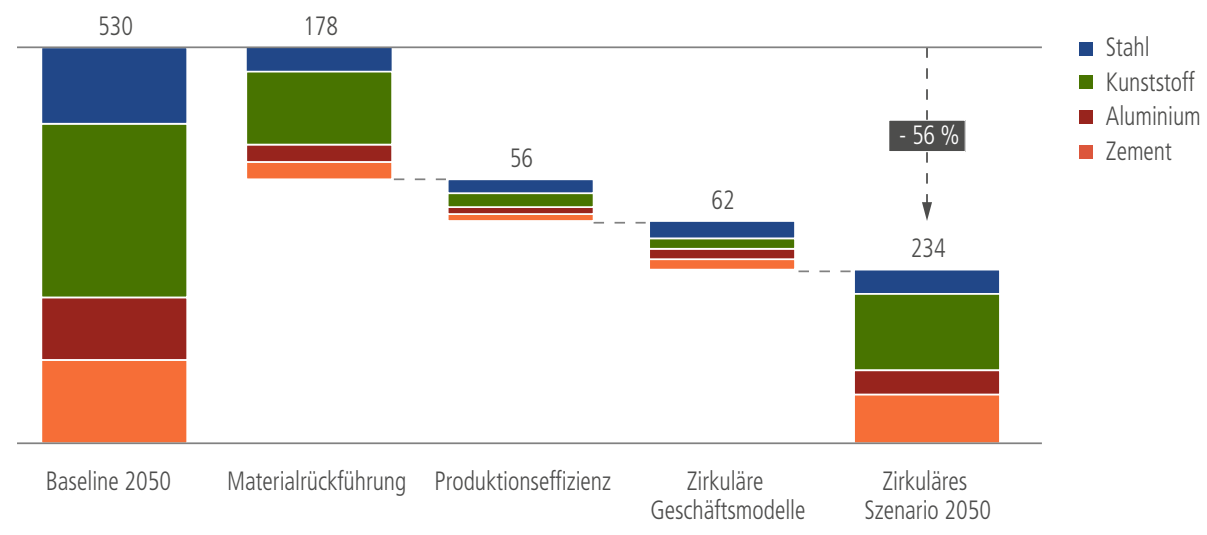

Abb. 13.2 CO2-Reduktionspotezial der Kreislaufwirtschaft am Beispiel der Schwerindustrien Stahl, Kunststoff, Aluminium und Zement in Europa (Mt $\mathrm{CO}_{2} / \mathrm{a}$ ). (Eigene Darstellung nach Material Economics 2020)

geht in Europa von einer Steigerung des BIP um weitere 0,5 Prozent und der Schaffung von ca. 700.000 neuen Arbeitsplätzen aus (European Commission 2020b).

Ob die Kreislaufwirtschaft diesen Erwartungen gerecht werden kann, wird sich zeigen und hängt von vielen Faktoren ab. Es kann jedoch davon ausgegangen werden, dass die Einführung einer Circular Economy wesentliche Reduzierungen der $\mathrm{CO}_{2-}$ Emissionen bewirken kann. Alleine in der Schwerindustrie hat die Einführung einer Circular Economy das Potenzial, bis 2050 etwa 56 Prozent der Emissionen gegenüber einem Basisszenario einzusparen. Betrachtet wurden die Stahl-, Kunststoff-, Aluminium- und Zementindustrie, welche derzeit die höchsten Emissionen aufweisen (s. Abb. 13.2). Über Maßnahmen wie Rezirkulation (mit 34 Prozent der größte zu erwartende Effekt), Effizienzsteigerungen bei Produkten und Materialien (elf Prozent) und neue Geschäftsmodelle im Sinne der Kreislaufwirtschaft (elf Prozent) sollen die Emissionen reduziert werden; insgesamt sollen diese Maßnahmen eine jährliche Reduktion von bis zu 296 Megatonnen $\mathrm{CO}_{2}$ bewirken (vgl. Material Economics 2020).

Im globalen Vergleich steht die EU aufgrund ihrer Bemühungen, schon in der Vergangenheit eine Circular Economy schrittweise herbeizuführen, relativ gut da und konnte auf diesem Entwicklungsweg eine Vorreiterrolle einnehmen. Richtet man den Blick jedoch auf die Einhaltung der globalen Klimaziele, steht auch die EU weiterhin vor großen Herausforderungen, wie das schon angeführte Beispiel der Schwerindustrien belegt. Die alleinige Einführung einer Circular Economy wird nicht ausreichen, die für diesen Sektor gesteckten Klimaziele bis zum Jahr 2100 zu erreichen, obwohl sie mit 


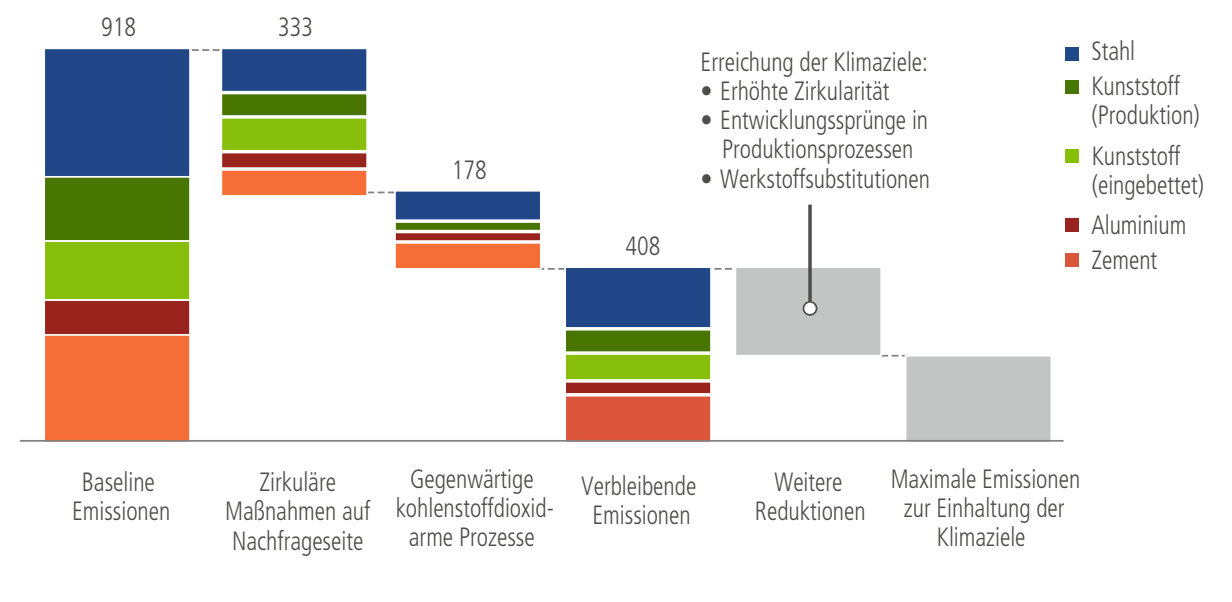

Abb. 13.3 Global kumulierte CO2-Emissionen in der Produktion von 2015 bis 2100 (Gt CO2). (Eigene Darstellung nach Material Economics 2020)

einem globalen Reduktionspotenzial von jährlich 333 Gigatonnen $\mathrm{CO}_{2}$ (s. Abb. 11.3) einen wesentlichen Beitrag dazu leisten kann (vgl. Material Economics 2020).

\section{Deutschland auf dem Weg zur Circular Economy}

Geprägt durch das Kreislaufwirtschaftsgesetz aus dem Jahr 2012 zählt Deutschland zu den Vorreitern der Kreislaufwirtschaft. Allerdings trifft dies lediglich auf die abfallwirtschaftliche Seite zu (Wilts 2016). Dass der Begriff der Kreislaufwirtschaft in Deutschland so eng mit der abfallwirtschaftlichen Seite verknüpft ist, hat historische Ursachen. 1972 wurde mit dem Abfallbeseitigungsgesetz erstmals in Deutschland das Abfallrecht in einem eigenen Gesetz zusammengefasst. Mit dem Abfallgesetz von 1986 wurden dann erstmals eine Reihe von Verordnungsermächtigungen aufgenommen, welche eine Durchsetzung von Abfallvermeidung und -verwertung auch auf Produktebene ermöglichten (Smeddinck und Klug 2016).

Zehn Jahre später, im Oktober 1996, wurde das Kreislaufwirtschafts- und Abfallgesetz erlassen. Durch dieses Gesetz wurde die Abfallwirtschaft in Deutschland maßgeblich verändert und erstmals der Begriff der Kreislaufwirtschaft eingeführt. Der Fokus lag jedoch weiterhin stark auf dem Umgang mit Abfall. Es wurden Hierarchien definiert, wonach die Abfallvermeidung der Abfallverwertung vorzuziehen war und diese wiederum der Abfallbeseitigung. Da dabei jeweils der umweltverträglicheren Verwertungsart der Vorzug zu gewähren war, richteten sich die Regelungen mehr als je zuvor auf die Förderung einer ressourcenschonenden Kreislaufwirtschaft (Komar 1998). 
Mit dem im Juni 2012 erlassenen Kreislaufwirtschaftsgesetz wurde das Ziel verfolgt, das bestehende deutsche Abfallrecht umfassend zu modernisieren. In den Vordergrund rückten zwar jetzt verstärkt die nachhaltige Verbesserung des Umwelt- und Klimaschutzes sowie der Ressourceneffizienz, jedoch lag der Fokus weiterhin auf der Abfallwirtschaft, da es vornehmlich um die Stärkung der Abfallvermeidung und des Recyclings von Abfällen ging (BMU 2020b).

Die Bundesregierung nahm die Veröffentlichung des Aktionsplanes "Den Kreislauf schließen" durch die EU-Kommission im Dezember 2015 zum Anlass, ihre Interpretation der Kreislaufwirtschaft erneut zu hinterfragen. Folgt man den Ausführungen des bestehenden Kreislaufwirtschaftsgesetzes, ist Kreislaufwirtschaft einer funktionierenden, effektiv regulierten Abfall- und Sekundärrohstoffwirtschaft gleichzusetzen. Der Aktionsplan der EU-Kommission zielt hingegen auf den Übergang in die Circular Economy ab. Darin eingeschlossen sind zahlreiche weitere Handlungsfelder wie Beschäftigung und Wachstum, Klima und Energie, die sozialpolitische Agenda, industrielle Innovationen, Produktdesign, Verarbeitung und nicht zuletzt Ressourceneffizienz und -schutz. Der EU-Aktionsplan betrifft Ressorts in der staatlichen Verwaltung, die weit über das bestehende Kreislaufwirtschaftsgesetz hinausgehen. Um diesem umfassenderen Ansatz auch im deutschen Sprachgebrauch gerecht zu werden, wurde in den vergangenen Jahren vom „Zirkulären Wirtschaften" oder von der „EU Circular Economy" gesprochen (Umweltbundesamt 2020).

Am 12.02.2020 hat nun die Bundesregierung einen Gesetzesentwurf zur Novelle des Kreislaufwirtschaftsgesetzes vorgelegt, der den notwendigen Entwicklungen, aber auch der Abfallrahmenrichtlinie der EU nachkommen soll. Drei Änderungen, die in der Novelle umgesetzt werden sollen, sind hervorzuheben: Zum einen sollen bei der Beschaffung der öffentlichen Hand bevorzugt Produkte ausgewählt werden, die rohstoffschonend, abfallarm, reparierbar, schadstoffarm und recyclingfähig sind. Zum anderen wird mit der Obhutspflicht ein weiteres Element der Produktverantwortung eingeführt, das Hersteller:innen und Händler:innen verstärkt in die Pflicht nehmen soll, insbesondere hinsichtlich der Anforderungen an Rücknahme- und Rückgabepflichten, der Wiederverwendung, der Verwertung und der Beseitigung der nach Gebrauch der Erzeugnisse entstandenen Abfälle und der Kostenbeteiligung an der Reinigung der Umwelt. Um die für die Obhutspflicht notwendige Transparenz zu gewährleisten, beinhaltet die Novelle ebenfalls die Grundlage für eine Transparenzverordnung, welche derzeit vom Bundesumweltministerium erarbeitet wird (BMU 2020a). Somit ist Deutschland auf einem guten, aber weiterhin langen Weg in Richtung einer vollständigen Circular Economy. 


\section{Kreislaufwirtschaft im EU Green Deal}

Vor dem Hintergrund der Agenda 2030 wurde auf EU-Ebene 2015 der erste Aktionsplan für die Kreislaufwirtschaft verabschiedet. $54 \mathrm{Maßnahmen} \mathrm{sollten} \mathrm{dazu} \mathrm{beitra-}$ gen, nicht nur den Übergang Europas zu einer Kreislaufwirtschaft zu beschleunigen, sondern es sollte gleichzeitig die globale Wettbewerbsfähigkeit gesteigert und das Wirtschaftswachstum Europas gefördert werden. Dabei wurde zum ersten Mal ein systemischer Ansatz über gesamte Wertschöpfungsketten hinweg verfolgt.

Nach einem positiven Zwischenfazit im 2019 veröffentlichten Bericht der EU-Kommission, nach dem sämtliche Maßnahmen abgeschlossen oder in Durchführung seien, wurde der Aktionsplan im Rahmen des EU Green Deal neu aufgelegt. Auf Basis des bisher Erreichten wird jetzt der Fokus auf die Bereiche Design, Produktion und Abfallvermeidung gelegt. So sollen Rechtsvorschriften erarbeitet werden, die sicherstellen, dass in der EU verkehrende Produkte länger nutzbar, reparierbar, wiederverwendbar und recyclebar sind. Den Fokus legt der Aktionsplans auf die Branchen Elektronik und IKT, Batterien und Fahrzeuge, Verpackungen, Kunststoffe, Textilien, Bauwesen und Lebensmittel, da sie einen hohen Ressourcenverbrauch haben und ihre Kreislaufeigenschaften ausbaufähig sind. Zur Abfallvermeidung will die EU-Kommission ein europaweites Modell zur Abfalltrennung und Kennzeichnung prüfen (European Commission 2020a).

Der neue Aktionsplan ist ein wichtiger Grundstein für eine europaweite gemeinsame Positionierung zur Senkung des Abfallaufkommens und zur Verbesserung der Voraussetzungen für ein funktionierendes Kreislaufsystem. Allerdings ist der Aktionsplan hinsichtlich quantifizierbarer Ziele und Zeitleisten zur Umsetzung durch die Mitgliedstaaten noch weitestgehend offen bzw. unkonkret. So fehlen ergänzend zum Ziel, die Siedlungsabfälle bis 2030 zu halbieren, weitere Vorgaben oder monetäre Anreize für einen sinkenden Verbrauch von Ressourcen und Primärrohstoffen oder Deponierungsverbote für recyclingfähige Abfälle.

\section{Wo stehen wir in Europa?}

Um den Stand der Umsetzung von Kreislaufwirtschaftsansätzen in der EU und den einzelnen Mitgliedstaaten zu erfassen, wurde 2018 der EU-Überwachungsrahmen für die Kreislaufwirtschaft vorgelegt. Darin wurden in vier Kategorien zehn Schlüsselindikatoren definiert (s. Abb. 13.4).

Auch wenn die europaweit einheitlichen Indikatoren noch nicht lange existieren, so können doch für die meisten von ihnen bereits längerfristige Entwicklungen ausgewertet werden (alle folgenden Werte nach Eurostat 2020). Dabei sind bereits für einige Indikatoren positive Trends zu verzeichnen. So steigt beispielsweise die Recyclingrate für alle Abfälle EU-weit stetig an und liegt aktuell bei 57 Prozent. Auf die 
1.EU-Selbstversorgung mit Rohstoffen

Anteil einer Auswahl wichtiger Materialien (einschließlich kritischer Rohstoffe),

die in der EU verwendet und in der EU hergestellt werden.

2. Grüne öffentliche Auftragsvergabe

Anteil großer öffentlicher Aufträge in der EU mit Umweltauflagen.

3 a-c. Abfallaufkommen

Pro-Kopf-Aufkommen an Siedlungsabfällen,

Gesamtabfallaufkommen (ohne dominante mineralische Abfälle) je BIP-Einheit und im Verhältnis zum heimischen

Materialverbrauch.

4. Lebensmittelverschwendung

Menge erzeugter Lebensmittelabfälle.

5a-b. Recyclingraten insgesamt

Recyclingraten bei Siedlungsabfällen und allen Abfällen ohne dominante mineralische Abfälle.

6 a-f. Recycling/Verwertung für bestimmte Abfallströme

Recyclingrate insgesamt bei Verpackungsabfällen, Kunststoffabfällen, Verpackungsmaterial aus Holz, Elektro und Elektronik-Altgeräten, recycelten Bioabfällen pro Kopf und Verwertungsrate bei Bau- und Abbruchabfällen.

7 a-b. Beitrag von recyceltem Material zur Nachfrage nach Rohstoffen

Anteil von Sekundärrohstoffen am Gesamtmaterial-

bedarf - für spezifische Materialien und für die gesamte Wirtschaft.

8. Handel mit recycelbaren Grundstoffen

Ein- und Ausfuhren ausgewählter recycelfähiger

Rohstoffe.

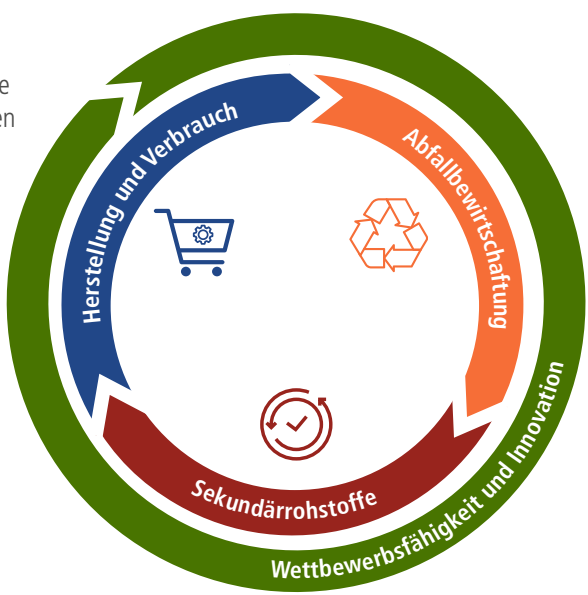

9 a-c. Private Investitionen, Arbeitsplätze und Bruttowertschöpfung

Private Investitionen, Anzahl Beschäftigte:r und Bruttowertschöpfung mit Bezug zu Bereichen der Kreislaufwirtschaft.

10. Patente

Anzahl der Patente im Zusammenhang mit

Recycling und Sekundärrohstoffen.

Abb. 13.4 Überwachungsrahmen für die Kreislaufwirtschaft. (Eigene Darstellung nach Europäische Kommission; https://ec.europa.eu/eurostat/de/web/circular-economy/indicators, zuletzt geprüft am 10.07.2020.)

einzelnen Abfallströme verteilt ergeben sich allerdings große Unterschiede. Während etwa bereits mehr als 80 Prozent organischer Abfälle und Bauschutt recycelt werden, liegt die Rate bei Elektroschrott oder Kunststoffverpackungen noch mit rund 40 Prozent $^{77}$ weit zurück. Die Recyclingrate von Siedlungsabfällen bewegt sich allerdings stetig auf die anvisierten Ziele zu (s. Abb. 13.5).

77 Genau: 38,8 Prozent Recyclingrate von Elektroschrott und 41,2 Prozent von Bauschutt. 


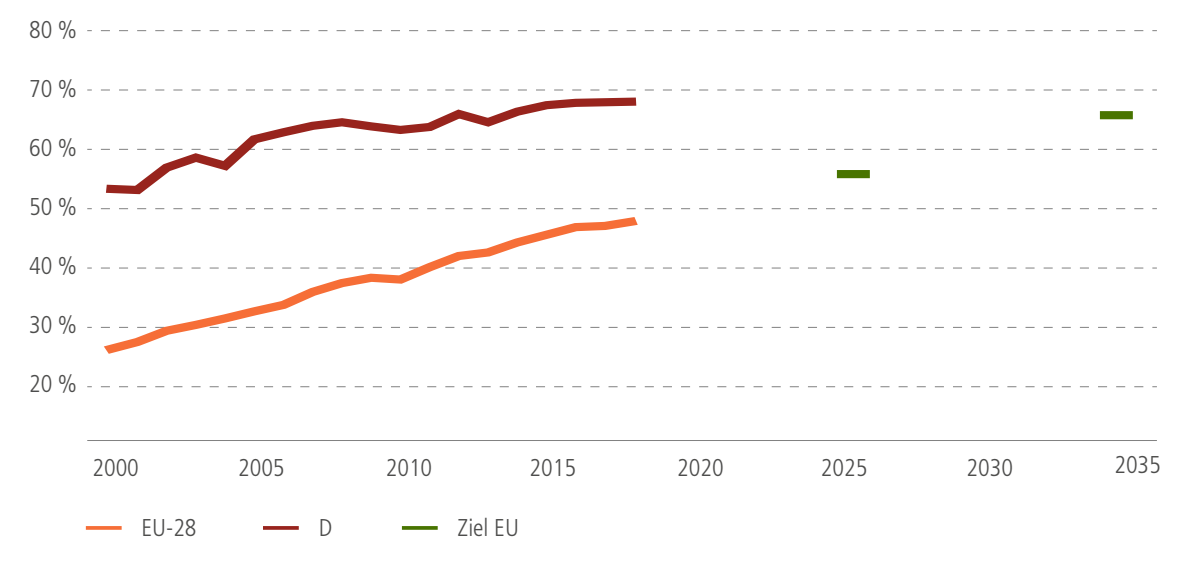

Abb. 13.5 Entwicklung der Recyclingraten von Siedlungsabfällen in EU-28 und Deutschland. (Eigene Darstellung nach Eurostat 2020)

Andere Indikatoren zeigen allerdings auch, dass die Entwicklung gerade in die falsche Richtung geht bzw. stagniert. So verzeichnet beispielsweise die Erzeugungsrate von Abfällen pro inländischem Materialverbrauch seit 2008 einen steigenden $\operatorname{Trend}^{78}$, die Nutzungsrate wiederverwendbarer Rohstoffe stagniert bei rund 11 Prozent und der Anteil der Beschäftigten in kreislaufwirtschaftsverbundenen Branchen verharrt seit 2013 bei gerade einmal 1,7 Prozent.

Wenig überraschend ist, dass sich zwischen den einzelnen Mitgliedstaaten erhebliche grundsätzliche Unterschiede in der Nachverfolgung entsprechender Umsetzungsmaßnahmen feststellen lassen ${ }^{79}$. Abgesehen davon sind die verfügbaren Daten aus einigen EU-Mitgliedstaaten teilweise noch sehr lückenhaft. Auch ein genauer Blick auf Deutschland zeigt, dass es sich in einigen Bereichen tatsächlich noch anstrengen muss, um seinen Ruf als Vorreiterstaat in Sachen Kreislaufwirtschaft zu verteidigen. Zwar sank das Aufkommen der Siedlungsabfälle entgegen dem EU-Trend seit 2016 geringfügig auf $615 \mathrm{~kg}$ pro Kopf in $2018 \mathrm{ab}$, die Recyclingquote für Abfälle insgesamt allerdings auch (zwischen 2010 und 2014 von 55 auf 53 Prozent). Hierbei zeigen sich insbesondere ein negativer Trend bei Verpackungen und organischen

78 Genau: Anstieg von 10,2 auf 12,8 Prozent zwischen 2008 und 2016.

79 Zum Beispiel steht die Recyclingrate von 83,3 Prozent für Verpackungen in Belgien einer Recyclingrate von 46,6 Prozent in Island gegenüber. Und während in Polen oder Spanien über 2 Prozent der Beschäftigten in kreislaufwirtschaftsnahen Sektoren arbeiten, sind es in Belgien oder den Niederlanden um die 1,1 Prozent. 
Abfällen sowie nur sehr zähe Verbesserungen beim Recycling von Elektroschrott (s. Kap. 8: Herausforderungen einer klimafreundlichen Energieversorgung). Auch im Bereich Wettbewerbsfähigkeit und Innovation muss Deutschland nachziehen. Zwar kann es unter den Mitgliedstaaten die höchste absolute Zahl an Beschäftigten in der Recyclingbranche vorweisen, bezogen auf die Gesamtbeschäftigten sind es aber gerade einmal 1,5 Prozent und die Bruttoinvestitionen in Sachanlagen liegen bei 0,1 Prozent des BIP. Hier kann über Fördermaßnahmen und klare regulative Ansätze noch einiges verbessert werden.

Förderung von Forschung und Entwicklung sind wichtige Treiber, um in der Umsetzung der Kreislaufwirtschaft weiterzukommen. Daher setzt die EU-Kommission auch im aktuellen Forschungsrahmenprogramm Horizon 2020 einen solchen Schwerpunkt. Eines der daraus hervorgegangenen Projekte ist CICERONE ${ }^{80}$. Das Projekt, dessen Akronym im Deutschen "Anleiter" oder "Fremdenführer" bedeutet, will Akteur:innen aus Forschung, Wirtschaft und Interessenverbänden mit entsprechenden Förderangeboten zusammenzubringen. Über die Plattform soll ein tieferes Verständnis zum Stand der Technik, der Kartierung der Interessenvertreter:innen, der bestehenden Forschungs- und Entwicklungsprioritäten sowie der Finanzierungs- und Rechtsmechanismen geschaffen werden. So können zum einen effiziente und zielführende Projekte auf den Weg gebracht sowie internationale und interdisziplinäre Zusammenarbeiten gefördert werden, zum anderen können die Förderprogramme entlang der tatsächlichen Bedarfe und Forschungslücken gestaltet werden (CICERONE 2020).

\section{Wie die Kreislaufwirtschaft gelingen kann}

Für die aktuellen Entwicklungen ist vor allem auf Seite der Produzent:innen kennzeichnend, dass neue Pfade von Pionier:innen ausgemacht werden, die jüngst durch Aktualisierung gesetzlicher Rahmenbedingungen auch wirksam unterstützt werden. Andererseits sind die Abfallquoten nach wie vor sehr hoch und Unternehmen verzichten noch immer überwiegend nicht auf schwer zirkulierbare Rohstoffe, beispielsweise bei der Verpackung. Vor diesem Hintergrund müssen Barrieren identifiziert werden, die Marktteilnehmenden in Deutschland und Europa - trotz verfügbarer Technologien sowie nachhaltiger Materialien und des Vorhandenseins eines Bewusstseins für Rohstoffknappheit - daran hindern, die R-Regeln (Reuse, Repair, Refurbish, Recycling, Rethink, Refuse, Reduce) konsequent umzusetzen.

Im Unterschied dazu zeigt sich Frankreich bereits seit Jahren sehr progressiv. So hat das französische Parlament Ende Januar 2020 ein umfassendes Gesetz gegen

80 Projektlangtitel: CIrCular Economy platfoRm for eurOpeaN priorities strategic agEnda. 
Verschwendung verabschiedet, das der Wegwerfgesellschaft ein Ende setzen soll. Hierunter fallen unter anderem Maßnahmen wie das stufenweise Verbot von Einwegplastik bis 2040 und neue Kennzeichnungen für Verbraucher:innen, die über die nachhaltige Produktion des Produktes informieren sollen. Ab 2021 soll ein Index von 1 bis 10 darüber Auskunft geben, welcher Arbeitsaufwand für die Reparatur eines elektronischen Produktes nötig ist. Auch soll über die Möglichkeit der Ersatzteilbeschaffung informiert werden. Der Kampf gegen Verschwendung und für eine solidarische Wiederverwendung wird ausgeweitet. Bereits seit 2016 sind größere Supermärkte dazu angehalten, nicht verkaufte Lebensmittel an soziale Einrichtungen zu spenden und nicht mehr zu vernichten. Ebenso sollen Telefon- und Internetanbieter Verbraucher:innen ab 2022 auf Treibhausgase aufmerksam machen, die durch digitalen Konsum entstehen (AHK Frankreich 2020). Die zum Teil sehr restriktiven Maßnahmen sind vielschichtig und zeigen verschiedene Wege auf, Teilhabende auf der gesamten Wertschöpfungskette zu sensibilisieren.

Im Grunde dreht sich alles in der Wirtschaft um die Sicherstellung grundlegender Bedürfnisse und Wünsche ("needs and wants"), die sich in einer entsprechenden Nachfrage widerspiegeln. Die Frage, ob ein bestimmtes Produkt oder eine bestimmte Dienstleistung ein Bedürfnis oder ein Wunsch ist, wird wohl niemals abschließend zu klären sein. So kann das, was die eine Person braucht, das sein, was eine andere Person haben will. Auch gibt es sicherlich eine Vielzahl von Möglichkeiten, um ein Bedürfnis oder einen Wunsch zu erfüllen. Letztlich sind aber die verfügbaren Ressourcen zur Erfüllung unserer Bedürfnisse und Wünsche begrenzt.

Zwischen Konsument:innen und Anbieter:innen besteht in der Wirtschaft eine komplizierte Wechselbeziehung und es ist zum Teil schwierig abzugrenzen, ob es sich beim Kauf eines Produktes um die tatsächliche Erfüllung eines originären Bedürfnisses bzw. Wunsches und somit um eine tatsächliche Nachfrage handelt, oder ob ein Kaufeffekt "künstlich" induziert wurde.

Entsprechend mannigfaltig können die Gründe für einen Austausch („Wegwerfen“) von Produkten, Dienstleistungen oder Services sein. Hier helfen der Begriff der Obsoleszenz (Ver-/Alterung oder Verschleiß) und die entsprechenden Gründe für den Austausch von Produkten (Dettli et al. 2014) bei der Analyse.

Obsoleszenz lässt sich grob in „absolute” Obsoleszenz (technisch möglichen Lebensdauer eines Produktes), "relative” Obsoleszenz (unabhängig von der technischen Lebensdauer des Produktes, sondern abhängig von der Entscheidung der Konsument:innen, das Produkt zu ersetzen) sowie "rechtliche" Obsoleszenz unterteilen (s. Abb. 13.6). 


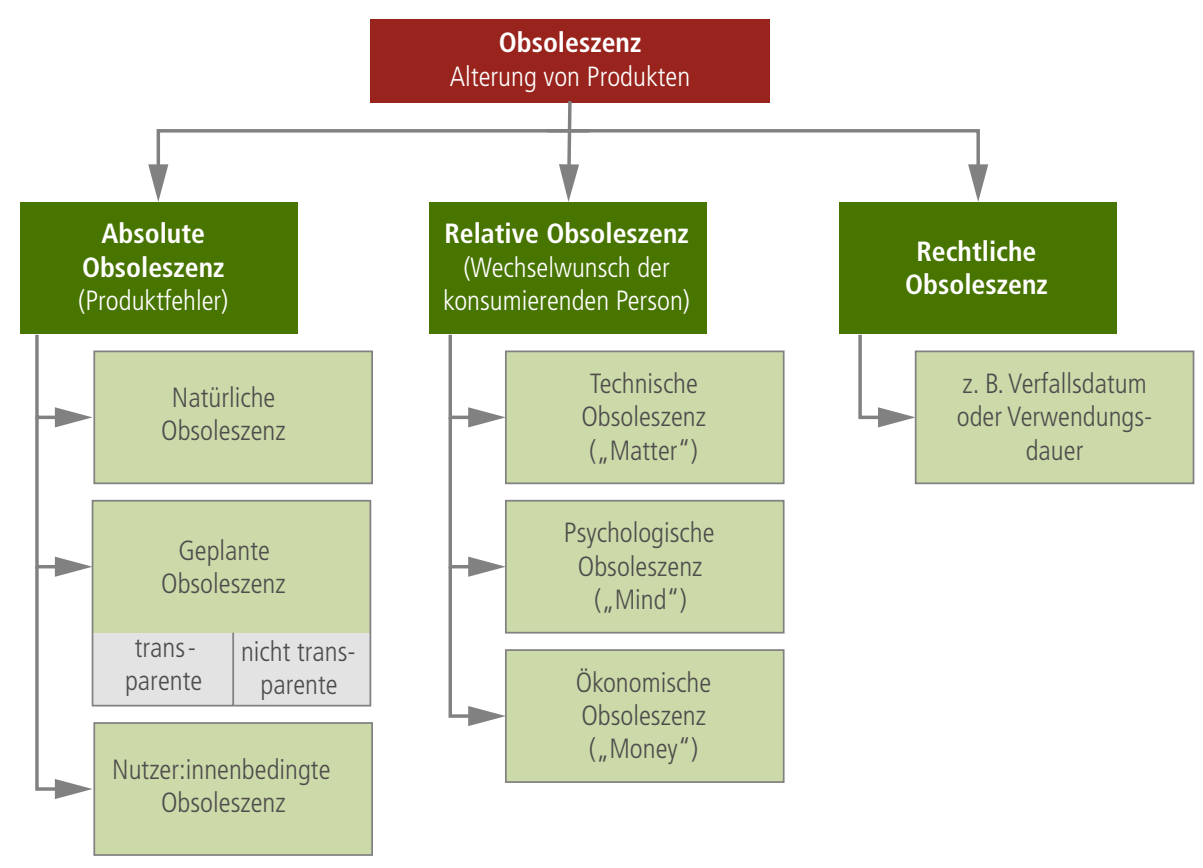

Abb. 13.6 Unterscheidung von absoluter, relativer und rechtlicher Obsoleszenz. (Eigene Darstellung nach Dettli et al. 2014)

\section{Absolute Obsoleszenz}

Wenn ein Produkt kaputtgeht, seine Funktion also nur noch begrenzt oder gar nicht mehr ausführen kann und somit das Ende der technischen Lebensdauer erreicht hat, spricht man von absoluter Obsoleszenz. Hierbei kann unterschieden werden in:

Natürliche Obsoleszenz - Alterung aufgrund nicht vermeidbarer material- und nutzungsbedingter Qualitätsverluste, welcher jedes Produkt unterliegt.

Geplante Obsoleszenz - angestrebte Lebensdauer eines Produkts, abgeleitet aus einer Vielzahl von Parametern wie der Nutzungsintensität, Exposition, Zielgruppe, Qualität, Funktionalität und/oder Status.

Geplante Obsoleszenz technischer Natur: Hier sind produktbezogene Aspekte dafür verantwortlich, dass ein Produkt nicht weiter genutzt werden kann (zum Beispiel Produktdesign, Schwachstellen bei der Komponenten- und Materialwahl, steigende Kompatibilitätsanforderungen bei Softwareinstallationen oder Fehlen von Ersatzteilen, die von einem Teilschaden zum Totalausfall führen). 
Geplante Obsoleszenz nicht technischer Natur: Unternehmensentscheidungen, die dazu führen, dass ein Produkt vorzeitig obsolet wird, um die Nutzungszeiten verkaufter Produkte zu verkürzen. Ein Produkt wird so unbrauchbar, weil die Kosten und der Aufwand es zu reparieren oder instand zu setzen so hoch sind, dass Reparaturen im Vergleich zu einem Neukauf ökonomisch uninteressant erscheinen. Gründe hierfür sind beispielsweise schneller Preisverfall, reparaturunfreundliches Design, hohe Reparaturkosten und mangelnde Verfügbarkeit von Ersatzteilen, Werkzeugen und Reparaturdienstleistungen.

Nutzer:innenbedingte Obsoleszenz: wenn ein Produkt beispielshalber aufgrund mangelhafter Wartung vorzeitig kaputtgeht. Oftmals ist nicht das Produkt schlecht, sondern die mangelhafte Wartung oder schlechte Behandlung ist Grund für einen Teil- oder Totalausfall eines Produkts.

\section{Relative Obsoleszenz}

Diese Art der Veralterung umfasst alle Faktoren, die nicht daher rühren, dass Produkte ihre Funktion nicht mehr erfüllen, sondern von der bewussten Entscheidung, dieses Produkt nicht mehr nutzen zu wollen. Verschiedene Ergebnisse lassen vermuten, dass die absolute Obsoleszenz, also jene, die in der technischen Alterung von Produkten liegt, weniger Einfluss auf die Lebensdauer von Produkten hat, als die relative Obsoleszenz, die in der Entscheidung der konsumierenden Person liegt (Cooper 2004).

Technologische Obsoleszenz: wenn ein vorhandenes Erzeugnis, das noch funktioniert, infolge der Einführung eines Neuen veraltet, welches die Funktionen besser erfüllt (Bodenstein und Leuer 1981; Packard und McKibben 2011).

Psychologische Obsoleszenz: wenn ein Erzeugnis, das qualitativ und in seiner Leistung noch funktionstüchtig ist, als überholt bzw. verschlissen betrachtet wird (zum Beispiel Mode). Gesellschaftliche Phänomene wie Gruppenzwang oder Statussymbole spielen hierbei für den Neukauf von Produkten eine Rolle. Die Hersteller:innen wissen dies mit Marketingkampagnen zu unterstützen. Zudem locken sie gezielt mit tiefen und zum Teil verzerrten Preisen, um die Verbraucher:innen zu einem Neukauf bzw. Mehrkauf zu animieren (Röper und Marfeld 1976).

Ökonomische Obsoleszenz: wenn ein Erzeugnis, das qualitativ und in seiner Leistung noch funktionstüchtig ist, als überholt gilt, weil es in seiner Wertigkeit weniger begehrenswert erscheint.

\section{Obsoleszenz aufgrund gesetzlich geregelter Angaben}

Es besteht die Möglichkeit, dass es zur Nichtverwendung von Produkten kommt, wenn diese strenge Normen und Grenzwerte erfüllen müssen oder wenn beispiels- 
weise gesetzliche Bestimmungen die Angabe von Verfallsdaten verlangen. Dies geschieht meist aus versicherungsrechtlichen Gründen, insbesondere bei Lebensmitteln, Medikamenten, Kontaktlinsen und Kosmetikartikeln.

\section{Der lange Weg zum Paradigmenwechsel}

Für das Erreichen der Ziele der Kreislaufwirtschaft tragen alle Akteur:innen innerhalb von Produktlebenszyklen und entlang von Materialwertschöpfungsketten Verantwortung, denn unsere Gesellschaft muss zu einer absoluten Reduktion des Verbrauchs nicht erneuerbarer Rohstoffe und der Entkopplung des Wirtschaftswachstums vom Rohstoffverbrauch kommen. Darum gilt es, unter anderem junge Unternehmen zu unterstützen und zu fördern, die nicht nur die Nachhaltigkeit als sinnvolles Betätigungsfeld sehen, sondern auch realisiert haben, dass die Kreislaufwirtschaft ein enormes wirtschaftliches Potenzial aufweist. Auch müssen Konsument:innen Verantwortung übernehmen und ihre individuelle Kaufentscheidung stärker an Nachhaltigkeitskriterien ausrichten. Dafür müssen sie aber hinreichend informiert sein. Somit ist es wiederum notwendig, dass Hersteller:innen ihrer Verantwortung nachkommen, eine hinreichende Transparenz beispielsweise in Bezug auf Lebensdauer, Lieferbarkeit von Ersatzteilen, Reparaturkosten, Herstellungsbedingungen und ökologische Vorteile von langlebigen Produkten herzustellen (PBnE 2017). Und die Übernahme von Verantwortung der Marktteilnehmer:innen muss auch, wenn sie nicht hinreichend wahrgenommen wird, rechtlich sichergestellt und sanktioniert werden.

Wie eingangs erwähnt, haben umfangreiche und über längere Zeit angewandte techno-ökonomische Paradigmen die Eigenheit, dass sie nur sehr schwer schnell und nachhaltig durch neue, effizientere ersetzt werden können. Dies muss jedoch geschehen, um die Klimaziele zu erreichen, die $\mathrm{CO}_{2}$-Emissionen zu reduzieren, die Vermüllung des Planeten zu beenden und dessen Ressourcen für nachkommende Generationen zu schützen. Dieser Handlungsdruck ist in Politik, Wirtschaft und Gesellschaft bereits stark spürbar und innovative Ideen von Akteur:innen, die neue Pfade konstituieren, stellen das allgemeingültige Paradigma für alle sichtbar infrage. Dies wäre nach Dosi (1982) der erste Schritt zur Ablösung eines Paradigmas. Ob die Praxis der "R-Innovationen" in der Kreislaufwirtschaft tatsächlich ein Modell werden kann, das in möglichst allen Sektoren Anwendungen findet, bleibt jedoch nicht nur eine angebotsseitige Frage. Konsument:innen befinden sich hier ebenso in einem Lernprozess wie Produzent:innen. Beide Seiten müssen darin motiviert und begleitet werden, zu lernen, dass sich das Credo „Rethink" aus den fünf R nicht nur auf Kaufentscheidungen, sondern darüber hinaus auf den Wandel ihres Rollen- und Zielverständnisses bezieht. So werden Produzent:innen und Verbraucher:innen zu „Bewahrer:innen", die so wenig Material wie möglich gebrauchen und so viel Material wie möglich wiederverwerten. 
Die EU selbst ist sich im Großen und Ganzen ihrer Verantwortung bewusst. Mit dem Green Deal hat sie ehrgeizige Reformen angestoßen, auf denen in Teilen die Zukunft Europas basiert. Im nächsten Schritt müssen Ziele konkretisiert, Mitgliedsstaaten motiviert und auch Anforderungen an die Staaten verbindlich gemacht werden. Sicherlich ist der Green Deal nicht perfekt - aber ein guter Kompromiss. 


\section{Literatur}

AHK Frankreich (2020): Frankreichs Parlament verabschiedet Gesetz gegen Verschwendung. Verfügbar unter https://www. francoallemand.com/publikationen/anmeldung-vorstellungstudie-deutsche-unternehmen-in-frankreich-geschaeftslage-einschaetzungen-und-perspektiven-2018-2022/news/news-detail/frankreichs-parlament-verabschiedet-gesetzgegen-verschwendung, zuletzt geprüft am 25.05.2020.

Bening, Catharina R. / Blum, Nicola U. / Haupt, Melanie (2019): Eine nachhaltige Kreislaufwirtschaft ist mehrdimensional | Die Volkswirtschaft - Plattform für Wirtschaftspolitik. Verfügbar unter https://dievolkswirtschaft.ch/de/2019/07/bening-08-09-2019/, zuletzt geprüft am 05.05.2020.

BMU (2020a): Novelle des Kreislaufwirtschaftsgesetzes legt Grundlagen für weniger Abfall und mehr Recycling - BMU-Pressemitteilung. Verfügbar unter https://www.bmu.de/ pressemitteilung/novelle-des-kreislaufwirtschaftsgesetzes-legt-grundlagen-fuer-wenigerabfall-und-mehr-recycling/, zuletzt geprüft am 07.05.2020.

BMU (2020b): Eckpunkte des neuen Kreislaufwirtschaftsgesetzes. Verfügbar unter https:// www.bmu.de/themen/wasser-abfall-boden/abfallwirtschaft/abfallpolitik/kreislaufwirtschaft/eckpunkte-des-neuen-kreislaufwirtschaftsgesetzes/, zuletzt aktualisiert am 07.05.2020, zuletzt geprüft am 07.05.2020.

Bodenstein, Gerhard und Hans Leuer (1981): Obsoleszenz - ein Synonym für die Konsumgüterproduktion in entfalteten Marktwirtschaften. In: Zeitschrift für Verbraucherpolitik, Vol. 5, No. 1+2, pp.

CICERONE (2020): Projektseite CICERONE. CIrCular Economy platfoRm for eurOpeaN priorities strategic agEnda. Verfügbar unter http://cicerone-h2020.eu/, zuletzt geprüft am 11.05.2020.

Cooper, Tim (2004): Inadequate Life?Evidence of Consumer Attitudes to Product Obsolescence. In: J Consum Policy 27 (4), S. 421-449. DOI: https://doi.org/10.1007/s10603-0042284-6.

Deutscher Bundestag (2018): Schriftliche Fragen. Drucksache 19/4634. Köln: Bundesanzeiger Verlag $\mathrm{GmbH}$.

Dettli et al. (2014): Optimierung der Lebens- und Nutzungsdauer von Produkten. Verfügbar unter https://www.econcept.ch/de/projekte/optimierung-der-lebens-und-nutzungsdauervon-produkten/, zuletzt geprüft am 07.05.2020.

Dievernich, Frank E. P. (Hg.) (2012): Pfadabhängigkeitstheoretische Beiträge zur Zukunftsgestaltung. Unter Mitarbeit von V. Tiberius. Wiesbaden: Springer Fachmedien Wiesbaden (Zukunftsgenese. Theorien des zukünftigen Wandels).

Dosi, Giovanni (1982): Technological paradigms and technological trajectories a suggested interpretation of the determinants and directions of technical change. In: Research Policy 11 (3), S. 147-162.

European Commission (2020a): A new Circular Economy Action Plan. For a cleaner and more competitive Europe. Brüssel. 
European Commission (2020b): Neuer Aktionsplan für die Kreislaufwirtschaft. Verfügbar unter https://ec.europa.eu/commission/presscorner/detail/de/IP_20_420, zuletzt geprüft am 11.03.2020.

Eurostat (2020): Indikatoren für die Kreislaufwirtschaft. Verfügbar unter https://ec.europa.eu/ eurostat/de/web/circular-economy/indicators/monitoring-framework, zuletzt geprüft am 11.05.2020.

Garud, Raghu (2010): Path Dependence or Path Creation? In: Journal of Management Studies 47 (4), S. 760-774.

Holzinger, Hans (2020): Mehr Effizienz allein reicht nicht. In: Sepp Eisenriegler (Hg.): Kreislaufwirtschaft in der EU. Eine Zwischenbilanz. 1st ed. 2020. Wiesbaden: Springer Fachmedien Wiesbaden, S. 195-216. Verfügbar unter https://doi.org/10.1007/978-3-65827379-8_13, zuletzt geprüft am 13.08.2020.

Komar, Walter (1998): Neues Kreislaufwirtschafts- und Abfallgesetz - Abnehmender Deponierungsbedarf durch verstärkte Abfallvermeidung und -verwertung. In: Wirtschaft im Wandel 11 (11), 9-15. Verfügbar unter https://www.iwh-halle.de/fileadmin/user_upload/ publications/wirtschaft_im_wandel/11-98-4.pdf, zuletzt geprüft am 07.05.2020.

Material Economics (2020): The Circular Economy - a Powerful Force for Climate Mitigation - Material Economics. Verfügbar unter https://materialeconomics.com/publications/ the-circular-economy-a-powerful-force-for-climate-mitigation-1, zuletzt geprüft am 20.05.2020.

Packard, Vance; McKibben, Bill (2011): Waste Makers. Brooklyn: Ig Publishing. Online verfügbar unter unter http://gbv.eblib.com/patron/FullRecord.aspx?p=3028731.

PBnE (2017): Impulspapier zur Produktverantwortung im Kontext der Kreislaufwirtschaft. Verfügbar unter https://valerie-wilms. de/themen/verkehr-mobilitaet/mobilitaet-2050/volltext-mobilitaet-2050/article/impulspapier_zur_produktverantwortung_im_kontext_der_ kreislaufwirtschaft, zuletzt geprüft am 13.08.2020.

Perez, C. (2009): Technological revolutions and techno-economic paradigms. In: Cambridge Journal of Eonomics (34), S. 185-220.

Röper, Burkhardt und Rolf Marfeld (1976): Gibt es geplanten Verschleiß? Untersuchungen zur Obsoleszenzthese. Göttingen: Schwartz (Schriften der Kommission für Wirtschaftlichen und Sozialen Wandel, 137).

Rosa, H. (1999). Rasender Stillstand? Individuum und Gesellschaft im Zeitalter der Beschleunigung. In: Manemann, J. (Hrsg.). Jahrbuch politische Theologie. Befristete Zeit. Münster: Lit Verl., S. 151-176.

Smeddinck, Ulrich und Ann Christin Klug (2016): Vom Abfallrecht zur Kreislaufwirtschaft. Untersuchungen zur Entwicklung des Abfallrechts und zu Abfallvermeidungsprogrammen nach $\S 33$ KrWG. Halle an der Saale: Universitätsverlag Halle-Wittenberg (Hallesche Schriften zum Öffentlichen Recht, Band 26). Verfügbar unter http://api.vlb.de/api/v1/asset/ mmo/file/191cff13-668f-422f-a95c-d1e78162cfbb, zuletzt geprüft am 06.05.2020. 
Stahel, Walter (2020): Warum ein Haushalten in Kreisläufen unsere Wirtschaft revolutionieren könnte. In: Sepp Eisenriegler (Hg.): Kreislaufwirtschaft in der EU. Eine Zwischenbilanz. 1st ed. 2020. Wiesbaden: Springer Fachmedien Wiesbaden, S. 9-21. Verfügbar unter https://doi.org/10.1007/978-3-658-27379-8_2, zuletzt geprüft am 13.08.2020.

Umweltbundesamt: Earth Overshoot Day 2019: Ressourcenbudget verbraucht. Verfügbar unter https://www.umweltbundesamt.de/themen/earth-overshoot-day-2019-ressourcenbudget, zuletzt geprüft am 09.05.2020.

Umweltbundesamt (2020): Leitsätze einer Kreislaufwirtschaft. Verfügbar unter https://www. umweltbundesamt.de/publikationen/leitsaetze-einer-kreislaufwirtschaft, zuletzt geprüft am 06.05.2020.

Wilts, Henning (2016): Deutschland auf dem Weg in die Kreislaufwirtschaft? Bonn: FriedrichEbert-Stiftung, Abteilung Wirtschafts- und Sozialpolitik (WISO Diskurs, 2016, 06).

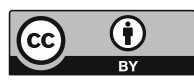

Dieses Kapitel wird unter der Creative Commons Namensnennung 4.0 International Lizenz http://creativecommons.org/licenses/by/4.0/deed.de) veröffentlicht, welche die Nutzung, Vervielfältigung, Bearbeitung, Verbreitung und Wiedergabe in jeglichem Medium und Format erlaubt, sofern Sie den/die ursprünglichen Autor(en) und die Quelle ordnungsgemäß nennen, einen Link zur Creative Commons Lizenz beifügen und angeben, ob Änderungen vorgenommen wurden.

Die in diesem Kapitel enthaltenen Bilder und sonstiges Drittmaterial unterliegen ebenfalls der genannten Creative Commons Lizenz, sofern sich aus der Abbildungslegende nichts anderes ergibt. Sofern das betreffende Material nicht unter der genannten Creative Commons Lizenz steht und die betreffende Handlung nicht nach gesetzlichen Vorschriften erlaubt ist, ist für die oben aufgeführten Weiterverwendungen des Materials die Einwilligung des jeweiligen Rechteinhabers einzuholen. 